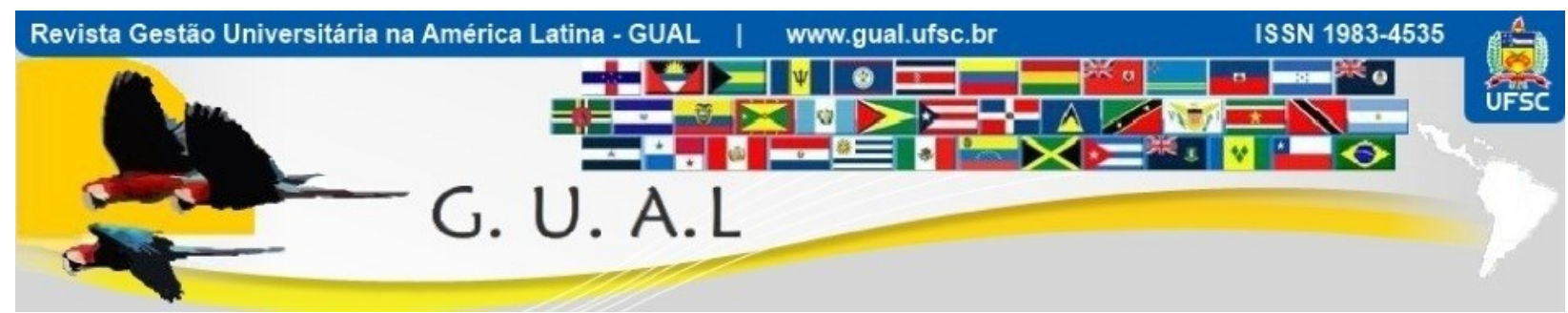

DOI: http://dx.doi.org/10.5007/1983-4535.2018v11n2p297

\title{
INTENCIÓN EMPRENDEDORA EN CONTEXTO UNIVERSITARIO BRASILEÑO
}

ENTREPRENEURIAL INTENTION IN THE BRAZILIAN UNIVERSITY CONTEXT

\author{
Roosiley dos Santos Souza, Doutora \\ Universidade Federal do Mato Grosso do Sul - UFMS \\ Universidade Nove de Julho - UNINOVE \\ roosiley@hotmail.com \\ Amelia Silveira, Doutora \\ Universidade Federal de Santa Catarina - UFSC \\ ameliasilveira@gmail.com
}

Recebido em 31/outubro/2016

Aprovado em 22/fevereiro/2018

Sistema de Avaliação: Double Blind Review

Esta obra está sob uma Licença Creative Commons Atribuição-Uso. 


\title{
RESUMÉN
}

El objetivo fue validar el Entrepreneurial Intention Questionnaire (EIQ ), como medida de la intención emprendedora en contexto universitario brasileño. El EIQ revisado por Liñán, Urbano y Guerrero (2011) toma como base la Teoría del Comportamiento Planeado (TCP), de Azjen (1991). La investigación consideró la Universidade Federal de Mato Grosso do Sul y la Universidade Federal da Grande Dourados, en el estado de Mato Grosso do Sul, Brasil. Cuantitativa y descriptiva, fue realizada con 505 alumnos de graduación de los cursos de Administración y de Ingeniería de Producción, en las dos universidades. El Modelaje de Ecuaciones Estructurales (MEE), con mínimos cuadrados parciales y modelo de caminos y el software SmartPLS 2.0 M3 dieron sustentación a los procedimientos estadísticos. Los constructos presentaron relaciones positivas y significantes explicando $13 \%$ de la varianza de la AP, $21,2 \%$ del CCP y 57,3\% de la IE. La relación entre la AP y la IE demostró la mayor fuerza, denotando el papel consolidado de la relación teórica. Los resultados indican que el EIQ fue validado, con prueba de las hipótesis, pudiendo ser considerado como modelo de medida para la intención emprendedora en el contexto de universidades brasileñas.

Palavras-chave: Intención Emprendedora. Entrepreneurial Intention Questionnaire (EIQ). Universidades Federales Brasileñas. Modelaje de Ecuaciones Estruturales (MEE). SmartPLS.

\begin{abstract}
The aim of the research was to validate the Entrepreneurial Intention Questionnaire (EIQ) as an instrument to measure entrepreneurial intention, in the Brazilian context. The EIQ as revised by Liñán, Urbano and Guerrero (2011) is based on Theory of Planned Behavior (TCP) by Azjen (1991). The research was quantitative and descriptive and considered the Federal University of Mato Grosso do Sul and the Federal University of Great Dourados, both in the state of Mato Grosso do Sul, Brazil. The survey was conducted with 505 undergraduate students in courses of Management and Production Engineering at the two universities. The Structural Equation Modeling (MEE), with Partial Least Squares Model, and software SmartPLS 2.0 M3 gave support to the statistical procedures. The constructs presented positive and significant relationship explaining $13 \%$ of the variance of the PA, $21.2 \%$ of BPC and $57.3 \%$ of IE. The relationship between PA and IE showed the greatest power among the observed denoting the strengthened role of the observed between PA and IE theoretical relationship. The results indicate that the EIQ was validated with test hypotheses, and can be considered as model for entrepreneurial intention in the context of the study of Brazilian federal universities.
\end{abstract}

Keywords: Entrepreneurial Intent. Entrepreneurial Intention Questionnaire (EIQ). Brazilian federal universities. Structural modeling Equations (MEE). SmartPLS. 


\section{INTRODUCIÓN}

Investigaciones han sido desarrolladas en contexto nacional e internacional con el propósito de verificar la disposición del individuo en emprender. En este sentido, la atención sobre el tema Intención Emprendedora ha crecido exponencialmente. Schlaegel y Koenig (2014), después de una revisión meta analítica de los determinantes de influencia en la intención emprendedora reconocerán como seminales los modelos de Shapero y Sokol (1982) y de Ajzen (1991). Y enfatizan que el estudio de Ajzen (1991) es lo que explica una cantidad mayor de variación en la intención, reconociendo su importancia en el contexto de este tema. De acuerdo con Lortie y Castrogiovanni (2015), la TCP de Ajzen (1991) se cambió en una de las teorías más utilizadas para explicar y predecir los comportamientos de los individuos. Esta teoría ha sido citada más de 5.000 veces, de acuerdo con la Web of Science, desde que Ajzen $(1988,1991)$ la publicó en un libro y en un artículo, respectivamente. Este artículo, en específico, ha servido de base para los estudios de intención emprendedora, en todo el mundo. Liñán y Fayolle (2015) constataron, además, que el artículo de Krueger y Carsud (1993) fue, probablemente, el responsable por hacer de la TCP la teoría de referencia en las investigaciones de intención emprendedora. Y, que el artículo de Autio et al. (2001) sirvió para establecer la aplicabilidad y utilidad del modelo TCP en emprendimiento, vinculado a los estudios de intención emprendedora. Ajzen (2006) presentó nuevas consideraciones conceptuales y metodológicas a respeto de la estructura de un cuestionario de TCP. Por su vez, estudios que utilizaron la TCP verificaron que, en media, las intenciones comportamentales explican $30-45 \%$ de la variación en las intenciones emprendedoras (KAUTONEN; VAN GELDEREN; FINK, 2013). Según Rueda, Moriano y Liñán (2015), la TPB (AJZEN, 1991, 2012) ha sido adoptada con suceso en estudios realizados a lo largo del tiempo para verificar el intento emprendedor de estudiantes universitarios.

Cabe llamar atención para otro punto convergente: las muestras de investigaciones que consideran como sujetos sociales los estudiantes de enseñanza superior. Y, también para el hecho de que las universidades se presentan como campo de investigación sobre este tema, cuando se trata de medir la intención de emprender de individuos jóvenes. Lima et al. (2014, p. 27), al estudiaren el escenario nacional, incluso, resaltan que los resultados presentados por el estudio GUESS Brasil reafirman la importancia de estudios realizados en el contexto de las universidades, sobre intención emprendedora, con estudiantes de graduación. Liñán y Chen (2009) afirman, aún, que una muestra de estudiantes universitarios es muy común en la 
investigación de la intención emprendedora. Y que estos sujetos sociales ofrecen la ventaja de edad y de cualificaciones semejantes, tornando la muestra más homogénea.

Hay convergencia, aun, cuanto a los modelos que se vuelven para explicar la relación entre las características personales del individuo y sus intenciones empresariales. Black (2012) presentó un cuadro comparativo de los modelos encontrados en la literatura, incluyendo las principales variables que cada uno de esos modelos contempla. Y, aquí, se puede identificar que el Entrepreneurial Intention Questionnaire (EIQ) emerge como un instrumento de colecta de datos - una escala psicosomática, capaz de medir la intención emprendedora en diferentes contextos culturales, una vez que en la literatura viene apuntando para la necesidad de un instrumento de medida padrón para investigar la intención emprendedora. De ese modo, la continuidad de los estudios en esa línea se hace necesaria y justifica, también, la necesidad de realización de nuevos estudios, para comprobación de la adecuación y utilidad del EIQ, como instrumento para mensuración de la intención emprendedora. Así en ese escenario son aún iniciales y recientes los estudios existentes, habiendo lugar y oportunidad para investigación que contribuya para la validación del EIQ, en organizaciones universitarias brasileñas. De esa manera el objetivo buscó validar la escala psicométrica propuesta inicialmente como Entrepreneurial Intention Questionnaire (EIQ), revista y modificada por Liñán, Urbano y Guerrero (2011) en el context universitario brasileño. Esto estudio tomó como base los constructos Actitud Personal (AP), Normas Subjetivas (NS), y Control del Comportamiento Percibido (CCP) en relación a la Intención Emprendedora (IE), propuestos en la TCP de Ajzen (1991).

El contexto de estudio consideró dos universidades federales brasileñas, situadas en el estado de Mato Grosso do Sul, Brasil, que presentan semejanzas cuanto a las variables organizacionales, como tamaño, complexidad organizacional, y tecnología, entre otras, siendo la Universidade Federal de Mato Grosso do Sul (UFMS) y la Universidade Federal da Grande Dourados (UFGD). Las dos ofrecen cursos de graduación en Administración y en Ingeniería de la Producción. Se justifica la elección de estas dos universidades federales en razón de que el estado de Mato Grosso do Sul es considerado nuevo, elevado a la categoría de estado en 1977, con fuerte ambiente emprendedor. Estas dos universidades están ubicadas geográficamente en municipios considerados emprendedores. Además sus estudiantes concordaron en participar de la investigación, respondiendo al Entrepreneurial Intention Questionnaire (EIQ), como instrumento de investigación para medir la intención emprendedora. 
Se ha estructurado este artículo en cinco partes. En la primera, introductoria, considero el tema, los principales autores y las teorías que consolidan el asunto, la justificativa para el interés de estudio, y el objetivo de investigación. La revisión de literatura se concentra en los autores de base y en los entendimientos principales sobre el modelo e instrumento a ser validado en la investigación. Aquí también se encuentran las hipótesis a ser probadas en el trabajo. En el ítem sobre métodos y las técnicas de investigación está garantizado el conocimiento sobre la trayectoria científica adoptada en los procedimientos de recolección de campo y de análisis de datos de la investigación. Los resultados describen el encontrado sobre la validación del EIQ y la prueba de las hipótesis, por medio de discusiones sobre el asunto. La conclusión sintetiza lo encontrado, buscando evidenciar cada punto del estudio, en el sentido de contribuir para ampliar la comprensión sobre intención emprendedora. Las referencias del que fue citado para amparar esta investigación constan al final del trabajo.

\section{REVISIÓN DE LITERATURA}

La Teoría del Comportamiento Planeado (TCP) propuesta por Ajzen (1991), es relativa al área de la psicología social. Es una teoría socio cognitiva que tuvo aplicación y suceso en varias áreas. El entendimiento es que, a la medida en que una persona tiene las oportunidades y los recursos necesarios, y tiene la intención de realizar el comportamiento, ella deberá tener suceso en hacerlo. Aún de acuerdo con Ajzen (1991, 2006), se deben considerar los constructos como actitud, norma subjetiva y control del comportamiento percibido para que haya la acción de emprender. De esa manera, "la teoría del comportamiento planeado postula tres determinantes conceptuales independientes de intención”, es decir, comprende tres variables independientes, que preceden la formación de la intención y que, por su vez, prevén el comportamiento. La primera variable es la actitud frente al comportamiento. Permite determinar el momento favorable para determinado comportamiento. La segunda variable corresponde a las normas subjetivas, que se refieren a la percepción que un individuo tiene sobre la comunidad que lo rodea, o el medio en que vive, y que puede influenciar su comportamiento. La tercera variable refleja el grado de percepción de control individual, y que induce el individuo a tener determinado comportamiento. (AJZEN, 1991). Además argumenta que las actitudes personales se refieren a la actitud o creencia delante de un comportamiento, y corresponden a la evaluación favorable o desfavorable que el individuo hace de ese comportamiento. Esta teoría procura explicar el 
comportamiento humano, en general, delante de una situación particular de intencionalidad. Las creencias normativas influencian las normas subjetivas, que también reflejan en la intención y comportamiento. Las creencias de control influencian el control comportamental percibido, que también refleja en la intención y comportamiento, sin embargo entre la intención y comportamiento hay un control de comportamiento real. De acuerdo con esta teoría es posible prever si un individuo va a crear un negocio en el futuro, analizando para el efecto, su intención emprendedora.

Liñán (2008) buscó probar y verificar la influencia de las variables do modelo de Ajzen (1991) - normas subjetivas, actitudes personales, control del comportamiento percibido y sus relaciones sobre la intención de emprender - en una muestra de 226 estudiantes de negocios y economía de la Universidad de Sevilla. El análisis factorial y el modelaje de ecuaciones estructurales fueron utilizados, siendo que el modelo explica 59,2\% de las intenciones emprendedoras. El Entrepreneurial Intention Questionnaire (EIQ) ha sido adoptado en esta investigación.

Liñán y Chen (2009) buscaron investigar las propiedades psicométricas de la construcción del cuestionario Entrepreneurial Intention Questionnaire (EIQ), para medir la intención emprendedora. Presentaron como resultados los análisis de confiabilidad y de validad realizados para evaluar la adecuación de este instrumento. Prueba la aplicabilidad del modelo de intención empresarial para diferentes contextos culturales: España y Taiwán. La muestra envolvió 387 alumnos universitarios, siendo que 71,9\% son estudiantes de negocios, y el restante son estudiantes de economía (26,8\%). Utilizó técnicas de ecuaciones estructurales, siguiendo la formulación de la Teoría del Comportamiento Planeado de Ajzen (1991). El primer paso fue utilizar el Alfa de Cronbach para probar la confiabilidad de las escalas propuestas; el segundo paso fue el análisis de validad; análisis factorial; la segunda parte fue probar el instrumento en culturas distintas. Como principales resultados los autores encontraron que las variables demográficas tienen pocos efectos significativos sobre los antecedentes de la intención emprendedora, siendo que las percepciones e intenciones son formadas de la misma manera, en Europa como en países Asiáticos. El modelo explica 55,5\% de la variación en la intención emprendedora, basada en Actitudes Personales y Control Comportamiento Percibido.

Con la continuidad de los estudios Liñán, Urbano y Guerrero (2011) reconocieran, sin embargo, que había algunos posibles problemas con el EIQ, como sesgo de aquiescencia. "aquiescencia es la tendencia de los individuos a concordar con las declaraciones en una 
escala o instrumento, y tiene sido tradicionalmente considerado como una característica de instrumento de medición usado."

Estos autores consideraron aún que:

[...] la solución más común es la construcción de escalas equilibradas, que generalmente son compuestas de ítems del tipo Likert. En una escala equilibrada todos los ítems son redactados de manera positiva; sin embargo, mitad de los ítems mide en una dirección del trazo en cuanto que la otra mitad es medida en la dirección opuesta (THOMPSON, 2009). La principal hipótesis de este tipo de medidas es que la aquiescencia de los ítems en una dirección será cancelada por aquiescencia para los ítems en la dirección opuesta. Así la suma de las puntuaciones de ítems apropiadamente invertidas (puntuación de contenido) es esperada para estar razonablemente libre de aquiescencia. (LIÑÁN; URBANO; GUERRERO, 2011, traducción nuestra).

Por esa razón, una versión de la escala psicométrica modificada fue desarrollada en la cual algunos ítems invertidos fueron incluidos. De esa manera, los autores esperaban minimizar la posible existencia de un problema estadístico. En este nuevo estudio Liñán, Urbano y Guerrero (2011) buscaron identificar algunos de los elementos cognitivos ambientales que pueden explicar las diferencias regionales en las intenciones emprendedoras. La muestra de conveniencia, con alumnos matriculados en el último año en Negocios y Economía, en España. La muestra final fue compuesta por 549 alumnos. En este estudio, Liñán, Urbano y Guerrero (2011) consideraron la necesidad de rever la escala psicométrica, teniendo verificado aquiescencia para algunos ítems de la escala psicométrica del modelo original de Liñán y Chen (2009). Así, la escala psicométrica fue revista, con cuestiones revertidas: Intención Emprendedora - IE (A4, A6, A9 - revertida, A13, A17 y A19 revertida); Actitud en relación al comportamiento personal - AP (A2 - revertida, A10, A12 revertida, A15 y A18); Control Comportamiento Percibido - CCP (A1, A5 - revertida, A7, A14, A16 - revertida, A20); Norma Subjetiva - NS (A3, A8, A11). El EIQ, en la integra, consta en el estudio de Liñán, Urbano y Guerrero (2011).

Naia (2013) intentó probar la TCP, de Ajzen (1991), en el ámbito de las ciencias del deporte, y así definir cuales variables influencian las intenciones emprendedoras de los alumnos de la Facultad de Motricidad Humana, en Lisboa. Con una muestra de 379 estudiantes, utilizó la escala psicométrica propuesta por Liñán, Urbano y Guerrero (2011), utilizando el análisis factorial confirmatorio y Modelaje de Ecuaciones Estructurales. Los resultados evidencian que la TCP es una teoría válida y que el instrumento de escala psicométrica desarrollado y ayustado por Liñán, Urbano y Guerrero (2011) puede ser aplicado en contextos diferenciados. 
En Brasil, Couto, Mariano y Mayer (2010) aplicaron el cuestionario de Chen y Liñán (2009) en un muestreo no probabilístico de estudiantes del curso de Administración de Empresas de la Universidade Federal Fluminense, en 2009. Fueron colectados 254 cuestionarios. Para compilar las informaciones colectadas, utilizaron el software CSPro $4.0 \mathrm{y}$, después de eso, los datos fueron trasportados para el SPSS 16.0, para el análisis de los resultados. El análisis factorial exploratorio fue utilizado como medio de evaluar si las respuestas de los estudiantes brasileños reflejarían la estructura conceptual del modelo de intención emprendedora (LIÑÁN; CHEN, 2009). Los resultados provenientes de la aplicación del EIQ no alcanzaron índices suficientes de confiabilidad y validad, no siendo posible, así, identificar la estructura del modelo de intención de emprendedora o una fuerte correlación entre los ítems que se refieren a los factores del modelo. Los autores consideraron como posibles causas para este resultados que las "características demográficas de cada población tienen fuerte influencia en las percepciones y sobre emprendimiento, $\mathrm{y}$, consecuentemente, de la intención emprendedora, por razones históricas, socioeconómicas o culturales." (COUTO; MARIANO; MAYER, 2010, p. 11).

Hecke (2011) realizó investigación para identificar distinciones significantes entre los alumnos en Administración y Ciencias Contables de las instituciones de enseñanza superior de Curitiba, PR. Para tanto, tomó como base la Teoría del Comportamiento Planeado (TCP) y el estudio de Liñán y Chen (2009). La muestra fue del tipo no probabilística, por conveniencia, adoptando el análisis factorial exploratorio para agrupar las variables analizadas y evaluar los constructos teóricos que fueron formulados. El análisis de regresión linear fue utilizado con el objetivo de verificar la influencia de cada uno de los constructos sobre la intención emprendedora. El autor observó que, en relación a las cuestiones que analizaban las actitudes personales de los respondientes, esas explican en hasta 47,2\% de las variaciones de las intenciones emprendedoras de los que están graduándose en Administración, y 60,9\% entre los estudiantes de Ciencias Contables.

Almeida (2013) verificó las relaciones entre valores, actitud en relación al emprendimiento y la intención emprendedora, en un estudio comparativo con universitarios de graduación en Administración, en las cinco regiones brasileñas y en Cabo Verde. El instrumento de investigación fue compuesto por un cuestionario sociodemográfico, la escala de Valores Humanos - Cuestionario de Perfiles de Valores de Schwartz (2001) y el Cuestionario de Intención Emprendedora (EIQ) de Liñán y Chen (2009). Los datos fueron tablados y analizados en lo software SPSS 21 y AMOS 21 para la producción de estadísticas 
descriptivas, análisis de confiabilidad, análisis de correlación, análisis de varianza (ANOVA), gráficos, análisis factorial confirmatoria y Modelaje de Ecuaciones Estructurales. Cuanto a las intenciones emprendedoras la muestra de mujeres presentaron menor intención emprendedora, actitud personal menos positiva en relación al emprendimiento y menor percepción de capacidad en iniciar una empresa, sin embargo indiquen poseer niveles semejantes de apoyo social (normas subjetivas).

Más recientemente, los estudios de Silveira et al. (2015), Sousa y Fontenelle (2015), Souza y Silveira (2016), Santos, Martins y Silveira (2016), entre otros, han sido presentados en el encuentro de la ANPAD, en los últimos dos años, siguiendo estas tendencias y denotando el interés por el tema.

\section{PROCEDIMIENTOS METODOLÓGICOS DE LA INVESTIGACIÓN}

La investigación sigue el dictamen de que una teoría es correcta si ella fuere pasible de comprobación con métodos científicos validados. De esa manera, el método adoptado es cuantitativo. Según Cooper y Schindler (2003, p. 136), este tipo de investigación está relacionado al empleo de recursos y técnicas estadísticas que permiten cuantificar los datos colectados. El método posee carácter inferencial. Intenta, con el apoyo en técnicas estadísticas, inferir generalizaciones derivadas de los datos observados (KERLINGER, 1980). Por su vez, Silveira et al. (2009, p. 69-70) complementan afirmando que estas investigaciones presentan el delineamiento predeterminado, estructurado, y la validad de la investigación ocurre por medio de la confianza en indicadores estadísticos. La investigación es transversal, ocurriendo en un período de tiempo específico. Con ese entendimiento, para su realización, el delineamiento adoptado fue el Modelaje de Ecuaciones Estructurales (MEE). Con aplicación en casi todas las áreas de estudio este se muestra adecuado, una vez que incluye administración, educación, psicología, sociología, entre otras (HAIR JÚNIOR et al., 2005, p. 466). Aún, de acuerdo con Hair Júnior et al. (2005, p. 475), la MEE, siendo un método confirmatorio, orientado más por teoría que por resultados empíricos, fornece al investigador la habilidad de acomodar múltiples relaciones de dependencia inter relacionadas en un solo modelo. Permite probar modelos teóricos en distintas áreas del conocimiento. Y, importante resaltar que en situaciones en que el investigador de las ciencias sociales aplicadas se depara con cuestiones de investigación que representan fenómenos en modelos “[...] con soporte teórico poco consagrado o aún poco explorado [...]” (RINGLE; SILVA; BIDO, 2014, p. 55, traducción nuestra), el uso de MEE basado en varianza o mínimos cuadrados parciales 
(PLS, Partial Least Square) es recomendado. También se entiende que el uso de ese enfoque es indicado cuando se observa una distribución de datos no adherente a la normalidad, y aun cuando el objetivo del estudio sea buscar una mayor predicción de las variables dependientes (HAIR JÚNIOR et al., 2014).

Debe ser resaltado que es de fundamental importancia que las medidas utilizadas en un estudio de MEE tengan sido desarrolladas a partir de estructuras teóricas que deberán generar las hipótesis del estudio, que ya tengan su validad demostrada, preferencialmente en estudios anteriores (HAIR JÚNIOR et al., 2014). En este estudio fue adoptado como software de MEE basado en varianza el SmartPLS (2005) (RINGLE; WENDE; WILL, 2015).

\subsection{MODELO DE INVESTIGACIÓN}

Así, el modelo delineado para mensuración de la intención emprendedora, que considera los constructos de Ajzen (1991), presentes en el QIE definido por Liñán, Urbano y Guerrero (2011) fue definido como sigue:

Figura 1 Representación del modelo de mensuración de la intención emprendedora

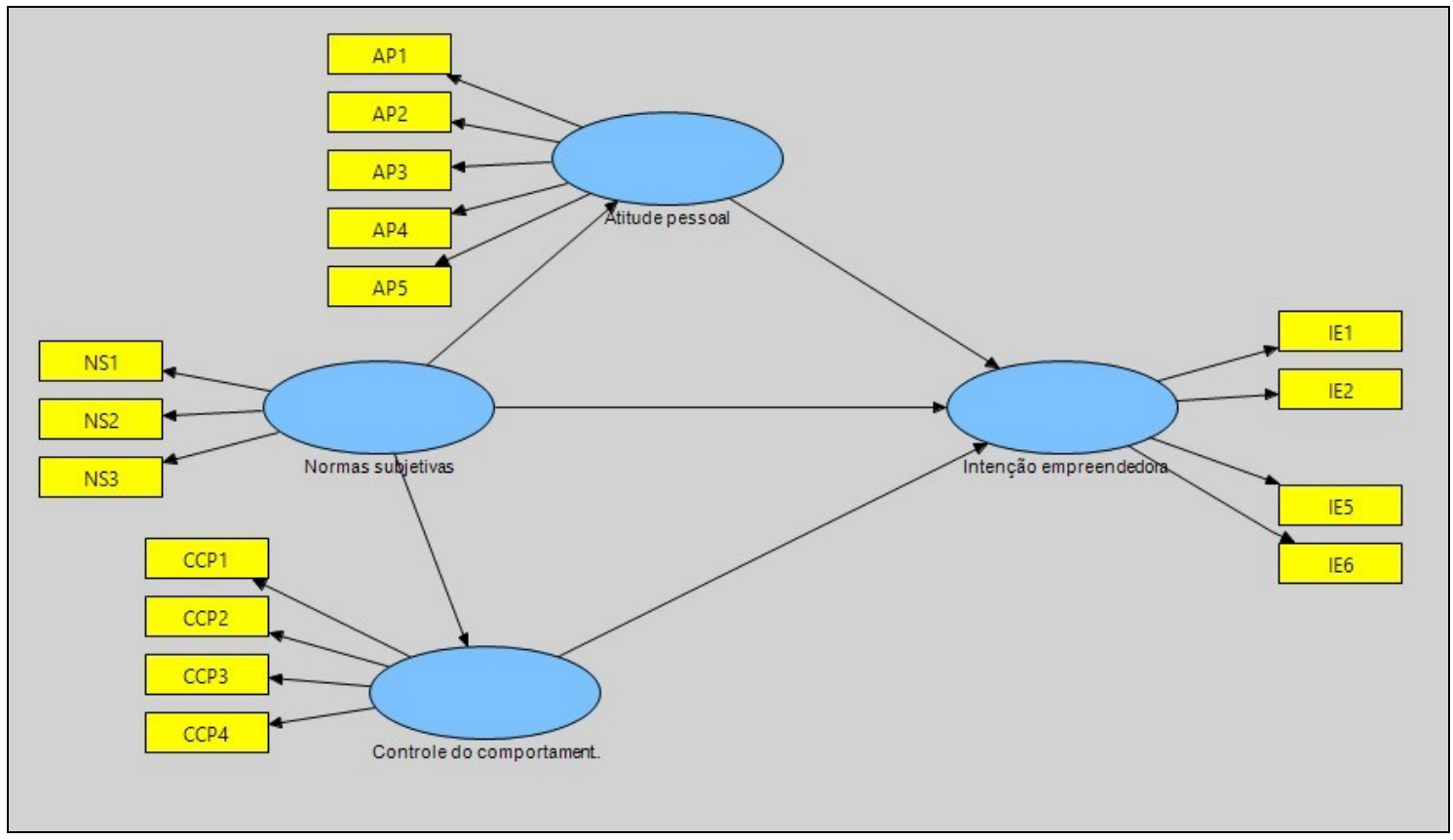

Fuente: Datos de la investigación obtenidos con la utilización del SmartPLS. 


\title{
3.2 HIPÓTESIS DE LA INVESTIGACIÓN
}

La hipótesis a ser probadas en este trabajo fueron definidas de la siguiente manera:

\begin{abstract}
H1: La Actitud personal tendrá una relación positiva y significante con la Intención emprendedora;

H2: El Control del comportamiento percibido tendrá una relación positiva y significante con la Intención emprendedora;

H3: Las Normas subjetivas tendrán una relación positiva y significante con la Intención emprendedora;

H4: Las Normas subjetivas tendrán una relación positiva y significante con la Actitud personal;

H5: Las Normas subjetivas tendrán una relación positiva y significante con el control comportamental percibido.
\end{abstract}

\subsection{PROCEDIMIENTOS PARA REALIZACIÓN DE LA INVESTIGACIÓN DE CAMPO}

Los procedimientos metodológicos adoptados para la realización de la investigación de campo se apoyan en Ringle, Silva y Bido (2014, p. 475), los cuales establecen algunas etapas para la aplicación de la MEE basada en varianza con el uso del SmartPLS. La primera etapa - de desarrollo de un modelo teórico - se basa en la observación del tamaño de la muestra necesaria para desarrollo de la mensuración de las relaciones causales propuestas con base en justificativas teóricas. El investigador debe determinar el tamaño del efecto $\left(\mathrm{f}^{2}\right)$ y fuerza del teste que busca para su estudio, observando la cantidad de relaciones causales (variables predictoras) de la variable dependiente con mayor número de predicciones. Para tanto, el investigador cuidadosamente define elementos básicos. El primer es la definición de constructo y sus variables de medición, siendo este un concepto teórico central utilizado para definir relaciones. En su terminología un constructo puede ser clasificado como exógeno o endógeno. Constructos exógenos, también conocidos como independientes, no son previstos, solamente son previsores de los constructos endógenos (dependientes). De esa manera se establece el modelo de mensuración por medio de las relaciones entre constructos. Desarrollase el modelo teórico en un diagrama de caminos (HAIR JÚNIOR et al., 2014) especificando el modelo estructural formalmente, en este se incluye la elaboración del instrumento de recolección de datos.

En la próxima etapa, el investigador busca la validad convergente del modelo elaborado (RINGLE; SILVA; BIDO, 2014). En esa etapa el investigador analiza los indicadores de Varianza Media Extraída (AVE) después del primero rodaje de los datos en el 
software. La validad convergente indica cuanto un modelo mensura "[...] la porción de los datos que es explicada por cada uno de los constructos o VL (variables latentes), respectivos a sus conjuntos de variables o cuanto, en media, las variables se correlacionan positivamente con sus respectivos constructos o VL [...]” (RINGLE; SILVA; BIDO, 2014, p. 62). Después de esa etapa, el investigador observará algunos indicadores de ayuste del modelo de consistencia interna, particularmente el Alpha de Cronbach y la Confiabilidad Compuesta. Estos indicadores evalúan "[...] se la muestra está libre de vieses, o, aún, si las respuestas - en su conjunto - son confiables [...]" (RINGLE; SILVA; BIDO, 2014, p. 63). En seguida se busca establecer la validad discriminante, o "[...] un indicador de que los constructos o variables latentes son independientes unos de los otros [...]" (RINGLE; SILVA; BIDO, 2014, p. 63). Esto es obtenido por medio de la observancia de la correlación de los ítems con sus respectivas variables y demás variables (crossloadings). También se observa la raíz cuadrada de la AVE de un constructo con los demás constructos. Después de este procedimiento ya se puede observar el $\mathrm{R}^{2}$, o coeficiente de determinación de las variables endógenas. Este indicador apunta la "[...] porción de la varianza de las variables endógenas que es explicada por el modelo estructural [...]" (RINGLE; SILVA; BIDO, 2014, p. 65). De otra manera, podemos decir que el $\mathrm{R}^{2}$ sugiere cuanto fue explicado de la variable dependiente por medio del modelo estructural elaborado.

Cuanto a los criterios de estimación, diversas tentativas han sido empleadas para evaluar intervalos de confianza, entre ellas Hair Júnior et al. (2005) sugieren el uso del método bootstrapping, donde la muestra original pasa a ser considerada como la población para fines de muestreo. El investigador debe entonces evaluar si las relaciones observadas en el modelo son significantes estadísticamente $(p<0,05)$, “[...] pues para los casos de correlación se establece la hipótesis nula (Ho) como $\mathrm{r}=0$ y para los casos de regresión se establece con Ho: $\Gamma=0$ (coeficiente de camino $=0$ ). Se $p>0,05$ se aceptan las Ho y se debe repensar la inclusión de VLs o VOs en el MEE [...]" (RINGLE; SILVA; BIDO, 2014, p. 65). En la secuencia son evaluados los indicadores de ayuste $Q^{2}$ y f ${ }^{2}$. El $Q^{2}$ mide la relevancia o validad predictiva del modelo elaborado, o de otra manera "[...] lo cuanto el modelo se acerca del que se espera de él [...]" (RINGLE; SILVA; BIDO, 2014, p. 68). El f² mide el tamaño del efecto, o por medio de un procedimiento de inserción y exclusión sistemática de cada constructo del modelo, se evalúa "[...] lo cuanto cada constructo es útil para el ayuste del modelo [...]" (RINGLE; SILVA; BIDO, 2014, p. 68). Para ayuste final del modelo se observa el Goodness of Fit - GoF, o calidad del ayuste. Este indicador es calculado con base en la 
“[...] media geométrica (raíz cuadrada del producto de dos indicadores) entre el $\mathrm{R}^{2}$ medio (adecuación del modelo estructural) y la media ponderada de las AVE [...]” (RINGLE; SILVA; BIDO, 2014, p. 69). En seguida, se analiza e interpreta los valores de los coeficientes de camino $(\Gamma)$. Estos indicadores sugieren la dirección y fuerza de la relación entre las variables del modelo. El sentido sugiere si la relación entre dos variables es directamente proporcional (coeficientes con señal positivo) o inversamente proporcional (coeficientes con señal negativo). La fuerza de la relación es observada por el indicador $t$ de student calculado anteriormente en la etapa de análisis de la significancia estadística de las relaciones estructurales (HAIR JÚNIOR et al., 2014). Concluyendo esa etapa el investigador puede examinar los resultados cuanto a su correspondencia con la teoría propuesta. Una ventaja de la adopción del MEE, en una investigación, es de que este utiliza variables manifiestas, no observables directamente, para representar un determinado constructo y, al mismo tiempo, permite que se disminuya el error de estimación de este constructo.

Conviene llamar la atención de que, al obtener datos en un levantamiento, el investigador debe estar enterrado del error de mensuración que puede ocurrir, en razón de la dificultad de comprensión, por veces, por parte del respondiente, sobre lo que le es preguntado, o hasta mismo por cuenta del propio concepto de utilizarse diversos ítems para mensurar una variable. Este error será incorporado al modelo probado, que establece las relaciones entre las variables teóricamente establecidas, y entonces la confiabilidad del modelo puede ser probada (HAIR JÚNIOR, 2010).

\subsection{INSTRUMENTO DE RECOLECCIÓN DE DATOS}

El instrumento de esa investigación se basa en el Entrepreneurial Intention Questionnaire (EIQ), con escala psicométrica con cuestiones revertidas, donde se presenta la escala siendo composta por cuatro bloques según su estructura original (LIÑÁN; URBANO; GUERRERO, 2011), después de traducido y adaptado para la lengua portuguesa. Además de esta parte, más dos bloques han sido presentados a los respondientes. En la primera parte, de opciones múltiples, los respondientes eligieron una entre las opciones listadas. Las cuestiones de ese bloque objetivan colectar datos sobre perfil sociodemográfico de los entrevistados cuanto al género, edad, estado civil, situación de trabajo actual, antecedentes emprendedores, participación en alguna capacitación emprendedora, renta propia y renta familiar. En la otra parte del cuestionario fue aún de interés de la investigación registrar los datos personales y 
profesionales de los alumnos que se mostraron interesados en continuar respondiendo investigaciones futuras sobre el tema.

\subsection{AMBIENTE DE LA INVESTIGACIÓN DE CAMPO}

Cabe destacar que la investigación fue realizada con todos los alumnos presentes en el período determinado para la recolección de todos los semestres de los cursos. Esa característica, en la recolección de datos, diferencia esta investigación de las demás que sirvieron de base teórica y que consideraban mayoritariamente estudiantes del último período de la graduación. Esa decisión de considerar todos los estudiantes ocurrió después del contacto con los coordinadores, que sugirieron que así fuese por consideraren que el intento emprendedor puede ser revelado de manera amplia. La recolección de los datos para esta investigación fue realizada en dos unidades de la Universidade Federal de Mato Grosso do Sul (UFMS) - en la ciudad de Campo Grande y en la ciudad de Três Lagoas - y en la Universidade Federal da Grande Dourados (UFGD), en la ciudad de Dourados. La muestra fue compuesta primordialmente por los alumnos presentes en el día de la aplicación del cuestionario, cuyo total fue de 524, siendo 362 de la UFMS (132 del curso de Administración y 230 del curso de Ingeniería de Producción) y 162 de la UFGD (122 del curso de Administración y 40 del curso de Ingeniería de Producción). Fueron distribuidos 524 cuestionarios, de los cuales 19 fueron considerados inválidos por presentaren datos faltantes. La muestra final fue de 505 respondientes.

\section{RESULTADOS DE LA INVESTIGACIÓN}

Inicialmente fue hecha la prueba a priori del tamaño de la muestra, utilizando el software $G^{*}$ Power, versión 3.1.9.2 y los valores del tamaño del efecto 0,15 (valor medio) y power (poder de la prueba) de 0,80, recomendados por Cohen (1988). De esa manera, el resultado del power (poder de la prueba) mostró que la muestra mínima seria de 77 cuestionarios. Sin embargo, en la investigación realizada se logró una muestra de 505 cuestionarios válidos, número superior al mínimo exigido. En relación a la escala psicométrica, así como ocurrió con los estudios de Liñán, Urbano y Guerrero (2011) y Naia (2013), en este estudio también fueron necesarios ayustes, como la exclusión de algunos ítems, para tanto, inicialmente fueron retirados ítems con carga inferior a 0,7, o aquellos con carga cruzada en otras variables distintas de sus respectivas dimensiones. Los ítems retirados pertenecen a los siguientes constructos: Intención Emprendedora y Control Comportamiento 
Percibido, con total de cuatro ítems. Investigaciones anteriores, como de Liñán, Urbano y Guerrero (2011) tuvieron ítems excluidos pertenecientes a los constructos del Control Comportamiento Percibido, Actitud Personal e Intención Emprendedora. Y en el estudio de Naia (2013) los ítems excluidos fueron los pertenecientes al constructo del Control Comportamiento Percibido y Actitud Personal. La diferencia en las exclusiones de los ítems en nuestro entendimiento se justificó por los aspectos relacionados a las variables exógenas pertenecientes a cada región donde los estudios fueron desarrollados. Después de la retirada de los ítems para ayuste del modelo se puede observar que la muestra siguió una distribución normal de los datos.

La Figura 2 presenta todos los indicadores del modelo estructural ayustado final y puede resumir los resultados obtenidos por medio del análisis de MEE.

Figura 2 Resultado del modelo estructural

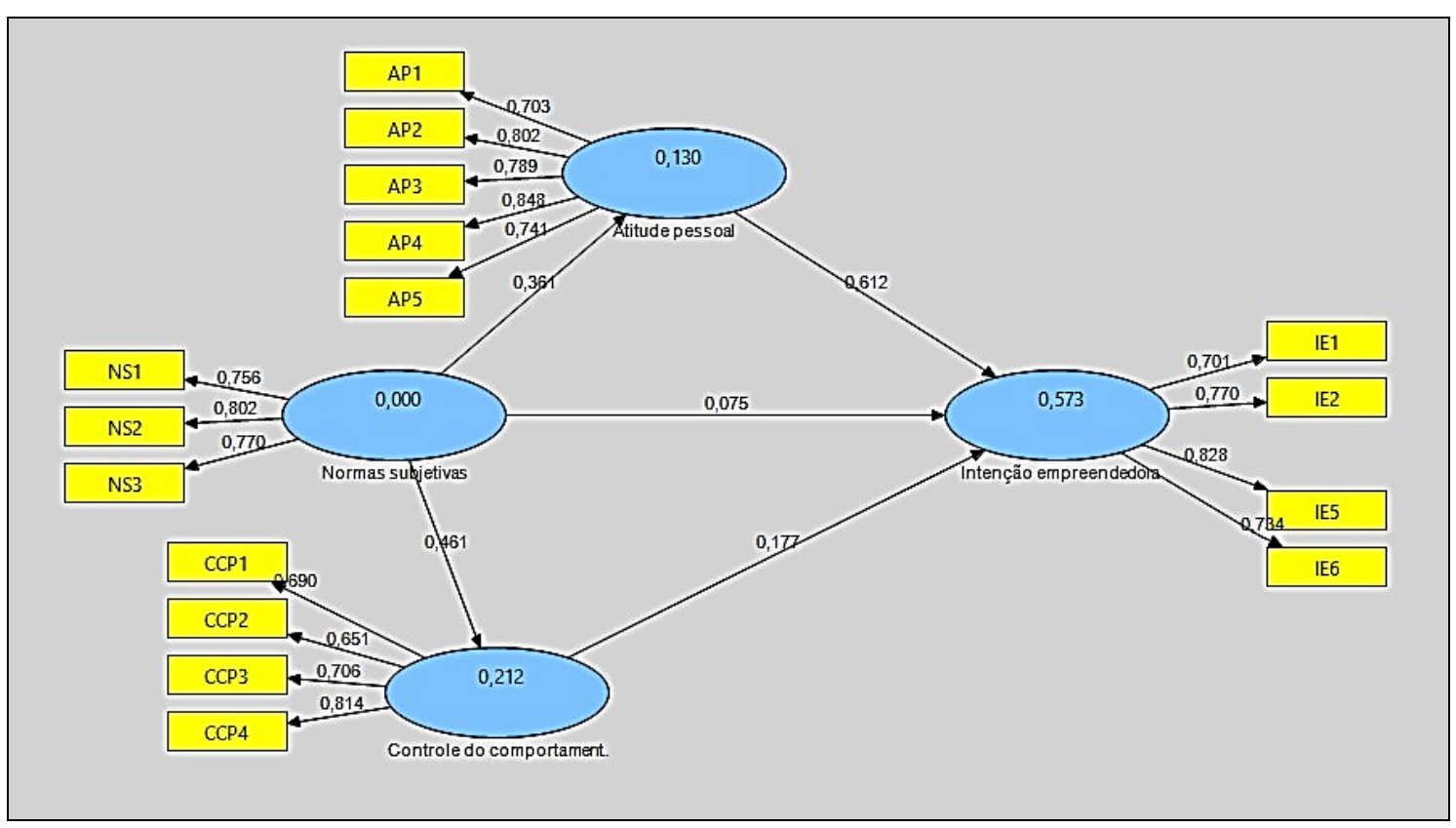

La variable con mayor poder de explicación en el modelo fue la Actitud Personal (AP), con $\mathrm{f}^{2}=0,408$, esto sugiere el papel central de la actitud en la explicación de la Intención Emprendedora (IE), como esperado (BAGOZZI, 1988; AJZEN; FISHBEIN, 2005). La variable latente (constructo) más explicada en el modelo fue la Intención Emprendedora (IE) $\left(\mathrm{Q}^{2}=0,309, \mathrm{R}^{2}=0,573\right)$. La relevancia predictiva del modelo observada por medio del $\mathrm{Q}^{2}$ evidencia como los coeficientes de camino mensuran de manera apurada determinada variable. Un mayor poder de explicación de esa variable, en relación a las demás se debe a 
que la IE antecede el comportamiento, y refleja que los investigadores en la muestra demuestran una predisposición bastante fuerte en emprender.

El modelo estructural ayustado presentó relaciones positivas y significantes $(\mathrm{p} \leq 0,05 \mathrm{y}$ $\mathrm{t}>1,96$ ) entre todos los constructos, identificados por el método de nuevo muestreo bootstrap, explicando 13\% de la varianza de la Actitud Personal (AP), 21,2\% del Control del Comportamiento Percibido (CCP) y 57,3\% de la Intención Emprendedora (IE). El modelo final presentó una buena calidad de ayuste. El cálculo del GoF, propuesto por Tenenhaus se quedó en 0,401 , por lo tanto, la recomendación de 0,36 como un valor mínimo en las ciencias sociales (WETZELS; ODEKERKEN-SCHRÖDER; OPPEN, 2009).

Todas las hipótesis fueron confirmadas demostrando el buen ayuste del modelo en el contexto de las instituciones de enseñanza investigadas. La relación entre la actitud y la intención $\left(\Gamma=0,612, \mathrm{t}_{(999)}=16,452, \mathrm{p}<0,001\right)$ demostró la mayor fuerza entre las relaciones observadas, denotando el papel consolidado de la relación teórica observada entre las actitudes e intenciones. Así, es posible afirmar, de manera resumida, que el modelo de mensuración final aceptó los criterios exigidos para buenas propiedades psicométricas, en relaciones positivas e significantes $(\mathrm{p} \leq 0,05$ y $\mathrm{t}>1,96)$ y que el modelo consiguió explicar 13\% de la varianza de la Actitud Personal (AP), 21,2\% del Control del Comportamiento Percibido (CCP) y 57,3\% de la Intención Emprendedora (IE).

El modelo estructural y la escala psicométrica propuesto por Liñán, Urbano y Guerrero (2011) se presentó adecuado en relación a los datos colectados en la UFMS y UFGD. Posibilitó proseguir con el avance de esa investigación, en el sentido de comprobar la adecuación de la aplicación de ese modelo y escala, en el contexto regional, considerando dos Instituciones de Enseñanza Superior (IES) públicas - UFMS y UFGD, para medir la intención emprendedora de los alumnos de graduación en Administración e Ingeniería de Producción.

La Teoría del Comportamiento Planeado de Azjen (1991, 2006), de la manera esperada, proporcionó soporte para las escalas psicométricas utilizadas en esa investigación, explicando las relaciones entre los constructos y representó un paso importante en el análisis de variables y en la validación de una escala psicométrica aplicable en distintos contextos culturales de previsión de las intenciones emprendedoras, presentadas en esa investigación.

\section{CONCLUSIÓN}

La conclusión cuanto al modelo de mensuración de la intención emprendedora delineado en este trabajo, así como las hipótesis definidas para comprobación conducen a un 
entendimiento básico y a una comprensión siguiente, conforme presentado en la secuencia, siguiendo el orden de los constructos presentes en la TCP (AJZEN, 1991), en el EIQ (LIÑÁN; URBANO; GUERRERO, 2011), y en las hipótesis definidas en este estudio.

Actitud Personal - de acuerdo con la literatura es el grado en que el individuo detiene una evaluación personal positiva o no en ser emprendedor. La investigación presentó el porcentual de $13 \%$, que significa que hay una evaluación positiva por parte de los alumnos en el sentido de que se vuelvan emprendedores. Es un porcentual bajo, sin embargo significa que el ambiente para el emprendimiento precisa ser más enfatizado, fortalecido, sea por parte de las universidades, o políticas públicas del propio gobierno del estado para atraer el público joven universitario.

La hipótesis H1: La Actitud Personal tendría una relación positiva y significante con la Intención Emprendedora - esa primera hipótesis fue probada y se observó que el constructo es fuerte y tiene un papel significante en el modelo. Se observó que en este estudio todas las cuestiones pertinentes al constructo fueron validadas, lo que no ocurrió en los estudios anteriores ya mencionados.

Normas subjetivas - ellas miden la presión social percibida de realizar o no comportamientos emprendedores. Remite para la percepción de que "las personas de referencia" aprobarán la decisión de volverse un emprendedor, o no. La investigación no registró ningún porcentual. En la literatura, las normas subjetivas no presentan datos satisfactorios en su gran mayoría, pero necesitan de atención por estar íntimamente vinculadas a las cuestiones sociales y económicas de una región. El estado de Mato Grosso do Sul es un estado relativamente nuevo si comparado con los estados de la propia región Centro Oeste y del Sudeste y Sur, sin embargo tiene condiciones de empezar un proceso de desarrollo de una cultura más emprendedora.

En relación a las hipótesis generadas a respeto del referido constructo, la hipótesis H3: Las Normas subjetivas tendrían relación positiva y significante con la Intención Emprendedora - a pesar de su bajo índice, la hipótesis fue confirmada.

Hipótesis H4: Las Normas subjetivas tendrían una relación positiva y significante con la Actitud Personal - hipótesis también fue confirmada.

Hipótesis H5: Las Normas subjetivas tendrían una relación positiva y significante con el control comportamental percibido. Esta hipótesis fue confirmada con índice elevado si comparado a la relación de la hipótesis anterior. El índice se muestra superior si comparado con la relación de los demás constructos. 
En relación a las normas subjetivas, es necesaria una reflexión sobre los condicionantes que las afectarían, como la cultura, la cual está íntimamente vinculada a las presiones producidas por el ambiente social, económico y político de la región.

Control del comportamiento percibido - es definido como la percepción de la facilidad o dificultad de volverse un emprendedor, la investigación registró porcentual de 21,2\%. Ese porcentual revela percepción de las dificultades y facilidades de ser emprendedor.

En relación a la hipótesis H2: El Control del comportamiento percibido tendría una relación positiva y significante con la Intención Emprendedora - fue confirmada, indicando que hay comportamiento emprendedor entre los alumnos de los cursos investigados en las dos IES.

Intención emprendedora - sufre influencia a partir de las actitudes, normas y comportamiento, registró en la investigación el porcentual de 57,3\%. Mismo con ausencia de porcentual de las normas subjetivas, el porcentaje muestra una tendencia para el desarrollo de comportamientos y actitudes que lleven el individuo a emprender. Aún de acuerdo con el modelo de Liñán y Chen (2009), el capital humano y las variables demográficas son los moderadores que influencian cada uno de los constructos - actitud personal, normas subjetivas y control del comportamiento percibido influenciando así las intenciones emprendedoras.

Importante resaltar que en esa fase de investigación, a pesar de la realización de la recolección de datos sobre las informaciones pertinentes a las variables demográficas, ellas no fueron utilizadas como soporte al modelo por no tener una escala psicométrica específica adoptada para tal, como ocurrió en el trabajo de Almeida (2013). Pues la propuesta de la investigación fue de contribuir para la validación del modelo y de una escala psicométrica orientada para intención emprendedora, una vez que la literatura en el área apunta para la necesidad de un modelo con una escala posible de ser aplicada en distintos contextos. Conforme recomendado por Almeida (2013, p. 346) deben ser desarrolladas investigaciones que consideren la diversidad de la población brasileña en lo que se refiere a sus valores, costumbres y las visiones de mundo.

Esta investigación tuvo como objetivo validar la escala psicométrica intitulada Entrepreneurial Intention Questionnaire (EIQ), y propuesta por Liñán, Urbano y Guerrero (2011). Esta escala tomó como base los constructos Actitud Personal, Normas Subjetivas, y Control del Comportamiento Percibido en relación a la Intención Emprendedora, propuestos 
en la Teoría del Comportamiento Planeado (TCP) de Ajzen (1991), para identificar la intención emprendedora.

La revisión de la literatura presentó los autores seminales que desarrollaron los modelos de intención emprendedora considerados como base para el estudio del tema. Y, los que se dedicaron a la definición de una escala psicométrica, intitulada Entrepreneurial Intention Questionnaire. Sin embargo, se evidenció que este aún no está plenamente validado.

El delineamiento metodológico adoptado en la investigación utilizando la MME como principal instrumento para estadísticamente analizar los datos, utilizando PLS-PM (Partial Least Squares - Path Model) posibilitó la validación del modelo. La utilización de la metodología de la MME permitió ayustar el modelo de selección en aquello que se juzgó necesario, como la exclusión de algunos indicadores con cargas factoriales muy bajas y que no comprometieron la validad del constructo. La metodología empleada para el análisis de los datos, utilizando el modelaje de ecuaciones estructurales con mínimos cuadrados parciales y modelo de caminos (PLS-PM), por medio del software SmartPLS, se mostró una técnica estadística de modelaje y análisis de datos adecuada en este estudio para validar un modelo aplicable en regiones y contextos diferenciados. Además, la prueba del papel mediador de la actitud de esos alumnos investigadores en relaciones a la intención emprendedora, en lo que se refiere a las relaciones con las normas subjetivas y con el control del comportamiento percibido, se volvió objeto de interés, posibilitando la proposición de más un aspecto al constructo de Liñán, Urbano y Guerrero (2011), proporcionando la ampliación del entendimiento cuanto a la mensuración de la intención emprendedora.

Por fin, buscando aún el mejoramiento de la escala EIQ, resta proponer la alteración del instrumento revisto por Liñán, Urbano Y Guerrero (2011), cuanto al papel mediador de la actitud en relación la intención emprendedora en sus relaciones con las normas subjetivas y con el control del comportamiento percibido. Esta se constituye en contribución más específica para el tema del estudio.

\section{REFERÊNCIAS}

AJZEN, I. Attitudes, personality, and behavior. Chicago: Dorsey, 1988.

AJZEN, I. Constructing a TPB questionnaire: conceptual and methodological considerations. Retrieved, March 22, 2006.

AJZEN, I. The theory of planned behavior. Organizational Behavior and Human Decision Processes, v. 50, n. 2, p. 179-211, 1991. 
AJZEN, I. (2012). The theory of planned behavior. En: VAN LANGE, P. A. M.; KRUGLANSKI, A. W.; HIGGINS, E. T. (Ed.). The handbook of theories of social Psychology. London: SAGE, 2012. p. 438-459.

AJZEN, I.; FISHBEIN, M. The influence of attitudes on behavior. En: ALBARRACÍN, D.; JOHNSON, B. T.; ZANNA, M. P. (Ed.). The handbook of attitudes. Mahwah, NJ: Erlbaum, 2005. p. 173-221.

ALMEIDA, G. O. Valores, atitudes e intenção empreendedora: um estudo com universitários brasileiros e cabo-verdianos. 2013. $400 \mathrm{f}$. Tese (Doctorado en Administración)-Fundação Getúlio Vargas, São Paulo, 2013.

AUTIO, E. et al. Entrepreneurial intent among students in Scandinavia and in the USA. Enterprise and Innovation Management Studies, v. 2, n.2, p. 145-60, 2001.

BAGOZZI, R. P.; YI, Y. On the evaluation of structural equation models. Journal of the Academy of Marketing Science, v. 16, n. 1, p. 74-94, 1988.

BLACK, M. M. Exploring the multi-focus influence of identity on students' entrepreneurial intent. 2012. Disponível em: $<$ https://shareok.org/handle/11244/7204>. Acesso em: 16 set. 2016.

COHEN, J. Statistical power analysis for the behavioral sciences. 2. ed. New York: Psychology Press, 1988.

COOPER, D. R.; SCHINDLER, P. S. Métodos de pesquisa em administração. 3. ed. Porto Alegre: Bookman, 2003.

COUTO, C. L. P.; MARIANO, S. R. H.; MAYER, V. F. Medição da intenção empreendedora no contexto brasileiro: desafios da aplicação de um modelo internacional. En: ENCONTRO NACIONAL DOS PROGRAMAS DE PÓS-GRADUAÇÃO EM ADMINISTRAÇÃO, 34, Rio de Janeiro, 2010. Anais... Rio de Janeiro: ANPAD. 2010. CD-ROM.

HAIR JÚNIOR, J. F. et al. Análise multivariada de dados. 5. ed. Porto Alegre: Bookman, 2005.

HAIR JUNIOR, J. F. et al. Primer on partial least squares structural equation modeling (PLS-SEM). Los Angeles: SAGE, 2014.

HAIR JUNIOR, J. F. Multivariate data analysis. 7. ed. Prentice Hall, 2010.

HECKE, A. P. A intenção empreendedora dos alunos concluintes dos cursos de graduação em administração e ciências contábeis das instituições de ensino superior de Curitiba-PR. 2011. 83 f. Disertación (Maestría en Ciencias Contables)-Universidade Federal do Paraná, Curitiba, 2011. 
KAUTONEN, T.; VAN GELDERENB, M.; FINK, M. Robustness of the theory of planned behavior in predicting entrepreneurial intentions and actions. Entrepreneurship Theory and Practice, July, 2013. DOI: 10.1111/etap.12056.

KERLINGER, F. N. Metodologia da pesquisa em ciências sociais. São Paulo: E.P.U., EDUSP, 1980.

KRUEGER, N.; CARSUD, A. Entrepreneurship intentions: applying the theory of panned behavior. Entrepreneurship \& Regional Development, v. 5, p. 316-323, 1993.

LIMA, E. et al. Educação Superior em Empreendedorismo e Intenções Empreendedoras dos Estudantes - Relatório do Estudo GUESSS Brasil 2013-2014. Grupo APOE - Grupo de Estudo sobre Administração de Pequenas Organizações e Empreendedorismo, PPGAUNINOVE. Caderno de pesquisa, n. 2014-03. São Paulo: Grupo APOE. 2014.

LIÑÁN, F. Skill and value perceptions: how do they affect entrepreneurial intentions? International Entrepreneurship and Management Journal, v. 4, n. 3, p. 257-272, 2008.

LIÑÁN, F.; CHEN, Y. Development and cross-cultural application of a specific instrument to measure entrepreneurial intentions. Entrepreneurship Theory and Practice, v. 33, n. 3, p. 593-617, May, 2009.

LIÑÁN, F.; FAYOLLE, A. A systematic literature review on entrepreneurial intentions: citation, thematic analyses, and research agenda. International Entrepreneurship Management Journal, 2013. DOI: 10.1007/s11365015-0356-5.

LIÑÁN, F.; URBANO, D; GUERRERO, M.. Regional variations in entrepreneurial cognitions: Start-up intentions of university students in Spain. Entrepreneurship \& Regional Development. v. 23, n. 3 e 4, p. 187-215, April 2011.

LORTIE, J.; CASTROGIOVANNI, G. The theory of planned behavior in entrepreneurship research: what we know and future directions. International Entrepreneurship and Management Journal, 4 March, 2015. DOI: 10.1007/s11365-015-0358-3

NAIA, A. M. P. Entrepreneurship education in sport sciences: implications for curriculum development. 2013. Tese (Doctorado en Ciencias de la Educación) -Universidade de LisboaFaculdade de Motricidade Humana. Lisboa, Portugal, 2013.

RINGLE, C. M.; WENDE, S.; WILL, A. SmartPLS 2.0 (2005). Disponible en: $<$ www.smartpls.de>. Accedido en: 02 diciembre. 2015.

RINGLE, C. M.; SILVA, D. da; BIDO, D.E. Modelagem de Equações Estruturais com Utilização do SmartPLS. Revista Brasileira de Marketing, May, 2014. DOI: 10.5585/remark.v13i2.2717

RUEDA, S.; MORIANO, J. A.; LIÑÁN, F. Validating a theory of Planned Behavior Questionnaire to measure entrepreneurial intentions. In: FAYOLLE, A.; KYRÖ, P.; LIÑÁN, F. Developing, shaping and growing entrepreneurship. Cheltenham, UK: Edward Elgar, 2015. p. 60-78. 
SANTOS, E. B. A.; MARTINS, F. S.; SILVEIRA, A. Intenção empreendedora: categorização, classificação de constructos e predição de modelo. In: ENCONTRO DA ANPAD, 40, Costa do Sauipe, 2016. Anais... Rio de Janeiro, ANPAD, 2016.

SCHLAEGEL, C.; KOENIG, M. Determinants of entrepreneurial intent: a meta-analytic test and integration of competing models. Entrepreneurship Theory and Practice. v. 38, n. 2, p. 291-332, 2014.

SHAPERO, A.; SOKOL, L. The social dimensions of entrepreneurship. In: Encyclopaedia of Entrepreneurship. Englewood Cliffs, NJ: Prentice-Hall, 1982.

SILVEIRA, A. et al. Intenção empreendedora dos participantes do Startup Weekend: o antes e depois da capacitação empreendedora. In: ENCONTRO DA ANPAD, 39, Belo Horizonte, 2015. Anais... Rio de Janeiro, ANPAD, 2015.

SILVEIRA, A. et al. Roteiro básico para apresentação e editoração de teses, dissertações e monografias. 3. ed. rev. aum. Blumenau, Edifurb, 2009.

SOUSA, A. M. R; FONTENELE, R. E. S. Dimensões individuais e ambientais na intenção empreendedora de estudantes universitários no Brasil. In: ENCONTRO DA ANPAD, 39, Belo Horizonte, 2015. Anais... Rio de Janeiro, ANPAD, 2015.

SOUZA, R. dos S.; SILVEIRA, A. Intenção empreendedora: validação do Entrepreneurial Intention Questionnaire (EIQ) em contexto brasileiro. In: ENCONTRO DA ANPAD, 40, Costa do Sauipe, 2016. Anais... Rio de Janeiro, ANPAD, 2016.

THOMPSON, E. R. Individual entrepreneurial intent: construct clarification and development of an internationally reliable metric. Entrepreneurship Theory and Practice, v. 33, n. 3, p. 669-694, 2009.

WETZELS, M.; ODEKERKEN-SCHRÖDER, G.; OPPEN, C. V. Using PLS path modeling for assessing hierarchical construct models: guidelines and empirical illustration. MIS Quarterly, v. 33, n. 1, p.177-195, 2009. 